\title{
THE CHEMICAL COMPOSITION OF POST AGB STARS
}

\author{
M. PARTHASARATHY \\ Indian Institute of Astrophysics, Bangalore 560034, India \\ P.GARCIA LARIO \\ Laboratorio de Astrofisica Espacial Y Fisica Fundamental, Estacion de Villafranca del \\ Castillo, Apartado de Correos 50727, E-28080 Madrid, Spain \\ and \\ S.R. POTTASCH \\ Kapteyn Astronomical Institute, Postbus 800, Nl-9700 AV Groningen, The Netherlands
}

The F-type supergiant HD 56126 (F5I) is an IRAS source with detached cold dust shell with characteristics similar to the dust shells around planetary nebulae. From an analysis of high resolution and high signal to noise ratio spectra metal and $\mathrm{CNO}$ abundances have been determined. It is found that in HD 56126 $[\mathrm{C} / \mathrm{H}]=-0.01,[\mathrm{~N} / \mathrm{H}]=+0.17,[\mathrm{O} / \mathrm{H}]=-0.02,[\mathrm{~S} / \mathrm{H}]=+0.01$ and $[\mathrm{Fe} / \mathrm{H}]<-1.0$. The $\mathrm{C} / \mathrm{N} / \mathrm{O}$ abundance ratios and $\mathrm{CNO}$ abundances relative to $\mathrm{Fe}$ of $\mathrm{HD} 56126$ and related post AGB stars suggest that they have on their photospheres the material processed by triple alpha, $\mathrm{CN}$ and $\mathrm{ON}$ cycles.

From the high resolution spectra we derive the radial velocity of HD 56126 to be $+105 \mathrm{~km} / \mathrm{sec}$, which suggest that HD 56126 is a high velocity star belonging to the old disk.

The abundance ratios $[\mathrm{C} / \mathrm{Fe}],[\mathrm{N} / \mathrm{Fe}],[\mathrm{O} / \mathrm{Fe}]$ and $[\mathrm{S} / \mathrm{Fe}]$ in $\mathrm{HD} 56126$ and related post AGB stars are large and cannot be explained by nucleosynthesis and or mixing. These large ratios are due to the depletions of Fe but not of $\mathrm{CNO}$ and $\mathrm{S}$ elements. The depletion of refractory elements and the lack of depletions in CNO and $\mathrm{S}$ in the atmosphere of these post AGB stars is similar to that observed in the interstellar medium. Since most of these stars have circumstellar dust shells, the depleted refractory elements appear to be locked up in dust grains. The results suggest that in the recent past (possibly during the OH-IR stars stage) the outer atmospheres of these star expanded and cooled to the limit of the condensation temperature of refractory elements. Formation of cores of dust grains very close to the stars and the resulting dust driven mass loss may be able to explain the observed abundance peculiarities in these post AGB stars.

Some of the post AGB stars such as BD +39 4926, HR 4049, HD 52961 and HD44179 are extremely deficient in $\mathrm{Fe}$ and most likely in other refractory elements. However their CNO, S and $\mathrm{Zn}$ abundances do not share this extreme deficiency. Why some of these stars show extreme deficiency of refractory elements while others do not suggests that some of them had very dense cool envelopes where dust formation was very efficient which has depleted the refractory elements by a large fraction. Or these stars have gone through several cycles of envelope expansion, cooling to the limit of condensation temperature of refractory elements and dust formation close to the star. There seems a process operating in these stars which has driven out only the dust but not the gaz making the photospheres with depleted refractory elements. 